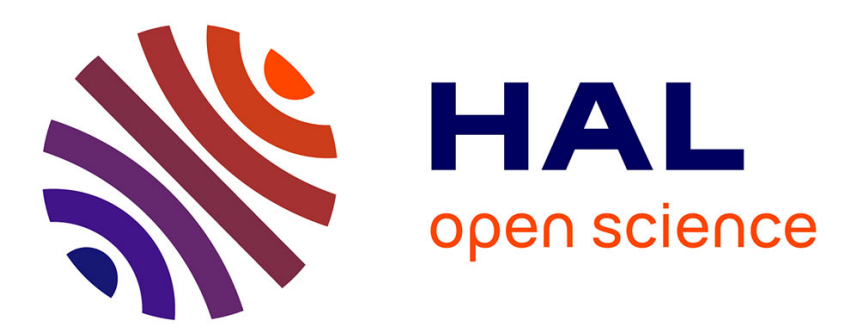

\title{
Mapping of 195 genes in cattle and updated comparative map with man, mouse, rat and pig
}

Hélène Hayes, C. Elduque, Mathieu M. Gautier, Laurent L. Schibler, Edmond E. Cribiu, Andre A. Eggen

\section{- To cite this version:}

Hélène Hayes, C. Elduque, Mathieu M. Gautier, Laurent L. Schibler, Edmond E. Cribiu, et al.. Mapping of 195 genes in cattle and updated comparative map with man, mouse, rat and pig. Cytogenetic and Genome Research, 2003, 102, pp.16-24. hal-02674251

\section{HAL Id: hal-02674251 \\ https://hal.inrae.fr/hal-02674251}

Submitted on 31 May 2020

HAL is a multi-disciplinary open access archive for the deposit and dissemination of scientific research documents, whether they are published or not. The documents may come from teaching and research institutions in France or abroad, or from public or private research centers.
L'archive ouverte pluridisciplinaire HAL, est destinée au dépôt et à la diffusion de documents scientifiques de niveau recherche, publiés ou non, émanant des établissements d'enseignement et de recherche français ou étrangers, des laboratoires publics ou privés. 


\title{
Mapping of 195 genes in cattle and updated comparative map with man, mouse, rat and pig
}

\author{
H. Hayes, C. Elduque, M. Gautier, L. Schibler, E. Cribiu and A. Eggen \\ Laboratoire de Génćtique biochimique et de Cytogénétique, INRA-CRJ, Jouy-en-Josas (France)
}

\begin{abstract}
Our on-going goal is to improve and update the somparative genome organization between cattle and man but also among the most detailed mammalian species genomes i.e. cattle, mouse, rat and pig. In this work, we localized 195 genes in cattle and checked all human/bovine non-concordant localizations found in the literature. Next, we compiled all the genes mapped in cattle, goat, sheep and pig $(2,166)$ for which the human ortholog with its chromosomal position is known, added corresponding data in mouse and rat, and ordered the genes
\end{abstract}

relatively to the human genome sequence. We estimate that our compilation provides bovine mapping information for about $89 \%$ of the human autosomes. Thus, a near complete, overall and detailed picture of the number, distribution and extent of bovine conserved syntenies (regardless of gene order) on human R-banded autosomes is proposed as well as a comparison with mouse, rat and pig genomes.

Copyright @2003 S. Karger AG, Basel
A major goal of livestock genomics is to map and identify genes involved in economically important traits and disease susceptibility and resistance. It requires powerful genome resources i.e. BAC and YAC libraries, radiation hybrid cell panels, detailed genomic maps and comparative maps. In recent years, human (International Human Genome Sequencing Consortium, 2001; Venter et al., 2001), mouse (Mouse Genome Sequencing Consortium, 2002) and rat (Rat Genome Project, 2003) genomes have been sequenced to near completion. This has provided an essential source of data for understanding genome organization and evolution, comparing genomes, identifying unknown genes, and analyzing gene expression and regulation. The cow is one of the economically most important species and over the past years, considerable work has been done to create detailed bovine gene maps but its

\footnotetext{
Received 28 May 2003; manuscript accepted 4 August 2003.

Request reprints from Hélène Hayes

Laboratoire de Génétique biochimique et de Cytogénétique

INRA-CRJ, 78352 Jouy-en-Josas cedex (France)

telephone: +33134652673 ; fax: +33134652478

e-mail: hayes@diamant.jouy.inra.fr

C. Elduque was a postdoctoral student in our laboratory for part of the work.
}

genome has yet to be sequenced. In this work, our aims were (1) to increase the number of genes mapped in cattle (195 new localizations), (2) to solve human/bovine non-concordant localizations currently found in the literature and (3) to update the organization of the cattle genome in relation to human, mouse, rat and pig.

\section{Materials and methods}

Primer pairs and probes for FISH mapping

Bovine YAC (Libert et al., 1993) and BAC clones (Eggen et al., 2001) were used to FISH-map 37 genes (see Table 3). They were obtained by PCR screening with three-dimensional pooling schemes as described in the respective publications using primer pairs listed in Tables 1 and 2. In addition, 151 caprine BAC clones isolated by Schibler et al. (1998) and corresponding to 151 genes (Table 3) were FISH mapped.

Chromosomal assignments on the INRA bovine $\times$ hamster somatic cell hybrid panel ( $\mathrm{SCH})$

Chromosomal assignments for seven of the genes (see Table 3) were obtained by PCR analysis of the INRA bovine $\times$ hamster hybrid panel as described by Laurent et al. (2000).

Fluorescent in situ hybridisation (FISH)

Chromosome preparations, DNA labelling, FISH, and R-banding are described in Hayes ct al. (1992, 2000). Chromosome and band numbering followed ISCNDB (2000). 
Table 1. List of genes localized with bovine YAC clones and corresponding primers. Thirty-five YAC clones were selected for 22 genes from human chromosomes 21 and 3 using specific primer pairs defined in conserved exons of the orthologous genes in man, cattle, sheep, pig, mouse and/or rat. Gene identity controls for bovine PCR products obtained with human or ovine primers revealed $88-100 \%$ sequence similarity. Note: 35 independent YAC clones were isolated but since 5 clones, each contained two genes, the total number in Table 1 amounts to 40 .

\begin{tabular}{|c|c|c|c|c|c|}
\hline Gene $^{a}$ & $\begin{array}{l}\text { Number of YAC } \\
\text { clones }{ }^{\mathrm{b}} \text { isolatcd and } \\
\text { hybridized/gene }\end{array}$ & Origin & $\begin{array}{l}\text { PCR } \\
\text { product } \\
\text { size (bp) }\end{array}$ & Forward primer & Reverse primer \\
\hline APP & 3 & bovine & 99 & GCAGAAGACGTGGGTTCC & CTTCAGCATCACCAAAGTTGA \\
\hline IFNARI & 1 & bovine & 290 & AGAAGTTTTCTGCGTCCTTTGCC & TGATGGTGGTATTCAGGTTCTTC \\
\hline TIAM] & 1 & human & 147 & GAGCCGAAAGGATTTCCTAAAG & GGCCTGGCCCCCTTCAGTG \\
\hline ATP50 & 2 & bovine & & AACCTGATCAATTTGCTTGCTGA & GTAACTGTGCATGGTACTTCTCCA \\
\hline GRIK1 & $3(1 \times n c)$ & human & 130 & GTTTTTCACCCTAATCATCAT & TGCCCCATATTCTATCTTG \\
\hline KCNE 1 & 3 & human & 79 & TGGGATTCTTCGGCTTCTTCA & GGGTCGTTCGAGTGCTCCAG \\
\hline SON & 3 & human & 104 & GCTGTAACCAACTTATCTAA & GAAATATTCTATCAGACCCTA \\
\hline SLC5A3 & $2(1 \times \mathrm{nc})$ & bovine & & AGGCTCTGCTCATGATCGTT & GGTTCCGCAACATTTTCAGT \\
\hline SOD1 & $2(2 \times n c)$ & bovine & 280 & GTTTGGCCTGTGGTGTAATTGGAA & GGCCAAAATACAGAGATGAATGAA \\
\hline PRSS7 & $2(1 \times n c)$ & bovine & 97 & ATTCAGCAAATGATAGATGAT & CCACTGGTCAAAGAGAAG \\
\hline POUlFl & 1 & bovine & 150 & GTAGTTTAACCCCTTGTCTTTAT & TTGGCTCTTCCACCAATTTACTT \\
\hline GAP43 & 1 (nc) & human & 203 & GATCCCAAGTCAAACAGTGTG & TCAGATGAACGGAACATTGC \\
\hline HES1 & $2(2 \times n c)$ & human & 153 & ATTGGCTGAAAGTTACTGTGG & GAGGTAGACGGGGGATTC \\
\hline KNG & 1 & bovine & 286 & CCTACTTCAGTTTTCTGAT & GAGATTACTAGCCCATTTTGGAA \\
\hline SIATI & 1 & bovine & & GCGTATTTTCCTGCTCAGAACAGC & CCGGGAGGACTTCAGAGATCCTG \\
\hline ERG & 2 & human & 295 & CACCAACGGGGAGTTCAA & CGCCACAAAGTTCATCTTCTG \\
\hline MX2 & 2 & ovine & 94 & GGCCCTGCATTGACCTCATC & GAGCTCTGGTCCCCGATAACG \\
\hline MXI & 2 & ovine & 94 & GGCCCTGCATTGACCTCATC & GAGCTCTGGTCCCCGATAACG \\
\hline RUNX1 & $2(1 \times \mathrm{nc})$ & human & 135 & CCTGTCGCCGTCTGGTAGGAG & GCTCATCTTGCCTGGGCTCAG \\
\hline TFF3 & 1 & human & 72 & GCGGCTACCCCCATGTCAC & ACACCAAGGCACTCCAGGGAT \\
\hline CBR1 & 2 & human & 125 & AGCAGAGGAAAGGGGACAAGA & GGCCAAGTACACAGGGGTCTC \\
\hline HMGN1 & 1 (nc) & human & 160 & TCTTGTACAATCCAGAGGAAT & AATAAACAACCAGCAAATGAT \\
\hline
\end{tabular}

a Gene symbols are from the HUGO Nomenclature database (http://www.genc.ucl.ac.uk/nomenclature/).

b $\mathrm{nc}=$ non chimeric bovine YAC clones.

Table 2. List of genes localized by FISH with bovine BAC clones (F) or by somatic cell hybrid mapping (SCH) and corresponding primers. Bovine BAC clones for 15 genes were isolated with primers defined from bovine or human sequences. Regions sharing a high sequence similarity with the orthologous human gene were determined using the Iccare program (T. Faraut; http://genopole.toulouse.inra.fr/Iccare). Gene symbols are from the HUGO Nomenclature database except when no official nomenclature is available to date as indicated by an asterisk.

\begin{tabular}{|c|c|c|c|c|c|c|}
\hline Gene & Origin & $\begin{array}{l}\text { GenBank } \\
\text { accession no. }\end{array}$ & $\begin{array}{l}\text { PCR } \\
\text { product } \\
\text { sizc (bp) }\end{array}$ & Forward primer & Reverse primer & $\begin{array}{l}\text { Mapping } \\
\text { procedure }\end{array}$ \\
\hline CCT8 & bovine & AF 136609 & 150 & GTGCCCTGGACTTGAACAGTA & CCAACGTTTTTATTTCCTTCTTG & $\mathrm{F}$ \\
\hline COLGAI & human & X99136 & 282 & TCTCCTCCCCGGCTGACATCACC & TCGTTGACGTCGGTGGCGTCGTTG & $\mathrm{F}$ \\
\hline SMP1* & human & NM_014313 & 102 & TTACCTTTAGCAACATAACCTC & TTGAAGGTGCTCGCATTTGGCT & $\mathrm{F}$ \\
\hline COP9* & bovine & AW464695 & 165 & AGCAGCAGTTAGCCAGACTCA & TCAGTAAAGTATGCCGAGGTGA & $\mathrm{F}$ \\
\hline COL6A3 & bovine & AW356722 & 200 & TCAATACCTACCCCAGCAAGA & GACCGCATCTAGGGACTTACC & $\mathrm{F}$ \\
\hline $\mathrm{CDC} 20$ & bovinc & BF193752 & 201 & ACATCCACCACCATGACGTT & CTTGATGCTGGGTGAAGGTCT & $\mathrm{F}$ \\
\hline IVL & bovine & AV618666 & 106 & CACACTCTGCCAGTGATTCAG & TGAAGTTGGCTTGCTTCACTT & $\mathrm{F}$ \\
\hline SSBP1 & bovine & BE 722675 & 100 & AGTTGCTCGGTCGAGTAGGTC & GATCGCCACATCTCATTTGTT & $\mathrm{F}$ \\
\hline HADHSC & bovine & BM481145 & 141 & ATGAGTTTGTGGCGAAGACC & ACTTGTCCAGCCTCTTGAACA & $\mathbf{F}$ \\
\hline VAV1 & bovine & BE485884 & 100 & CTACCAACAGAACTCTCTGAAGGA & TGATGGTTTGTTGATGGCTCT & $\mathbf{F}$ \\
\hline HSPA4 & bovine & $\mathrm{B} 1537416$ & 196 & TGACGAGTATCTGCAGCCCTA & TCAATGTCCATTTCAGGAAGC & $\mathbf{F}$ \\
\hline $\mathrm{RAB} 18$ & bovine & ВМ967938 & 168 & ACAAGGTCTGGTTTTGGCTCT & CATCCTAGGAAAGCTGTGGAA & $\mathbf{F}$ \\
\hline PSMA7 & bovine & BF045709 & 112 & CCTCACTGCTGATGCAAGAAT & GGCGATATAACGGGTGATGTA & $\mathbf{F}$ \\
\hline GNAO1 & bovine & BM286134 & 182 & CGACACCAACAACATCCAGTT & TGTCAAGGGCAGAGAGTGG & $\mathrm{F}$ \\
\hline SERPINO1 & bovine & AV606014 & 102 & TCACCATGGAGCAGCTGAG & AGGGGGAGATGAAGATGTTTC & $\mathrm{F}$ \\
\hline TRIM 17 & bovine & BE668983 & 176 & TCTTCCTGGATTTTGAAGCTG & CTATCCCTTCACCCACAGAGTC & $\mathrm{SCH}$ \\
\hline ELF2 & bovine & AW463485 & 104 & GCTGAGCCTTAACTTCCGAGT & CTGGCCTTTTGCTATAACACG & $\mathrm{SCH}$ \\
\hline IL6R & bovine & BE685522 & 121 & CAGGAGCCCTGCCAGTATT & ACTTGTTCCCGGCACTGTT & $\mathrm{SCH}$ \\
\hline RAB33B & bovine & AV594098 & 199 & CAATGACATGCTGGTGCTAAA & GTGTCACAGCGACAAACTTGA & $\mathrm{SCH}$ \\
\hline OSBPL8 & bovine & BE 665912 & 174 & CGGGTAACTCGAGCCATAAAT & TGCAAACTTGTAATGCCATTCT & $\mathrm{SCH}$ \\
\hline ARF1 & bovine & AV601289 & 147 & GACCTCCCTAATGCCATGAAC & GAGCTGATTGGACAGCCAGT & $\mathrm{SCH}$ \\
\hline SAG & bovine & J02955 & 189 & GTACGTGTCTCTGACGTGTGC & AGCAGGAAGGGGTAGGTGTT & $\mathrm{SCH}$ \\
\hline $\mathrm{ACK} 1$ & bovine & BG690194 & 194 & CTGTCTCCACAAGGCTCCAG & ACGATGGGCAAGATGCAG & F (unexpected) \\
\hline NDUFC1 & bovine & $\mathrm{X} 63214$ & 106 & CCGTAAAGTGATTATAGCAGTTCC & AAAACACAAATGCTGACTTGACA & $F$ (unexpected) \\
\hline NDUFV2 & bovine & M22539 & 157 & GCAGAAATTTTACAAGTACCTCCA & TCTGAATGGCTTCCAGTATGC & $\mathrm{F}$ (unexpected) \\
\hline
\end{tabular}


Table 3. List of new gene localizations on bovine chromosomes obtained by FISH and somatic cell hybrid mapping in this study. Normal characters FISH with caprine BACs, bold italic with bovine YACs, bold normal with bovine BACs, in shaded background somatic cell hybrid mapping.

\begin{tabular}{|c|c|c|c|c|c|c|c|c|c|}
\hline Gene & Localization & Gene & Localization & Gene & Localization & Gene & Localization & Gene & Localization \\
\hline KRTAP8* & $\operatorname{lq} 12.2$ & GDF 8 & $2 \mathrm{q} 12.2$ & CSN2 & $6 \mathrm{q} 32$ & EDNRB & $12 q 22$ & $\mathrm{C} 9$ & $20 q 17$ \\
\hline$A P P$ & $1 q 12.2$ & ENI & $2 \mathrm{q} 33$ & CSN1S1 & $6 \mathrm{q} 32$ & IL2RA & 13 q13med & SLC6A3 & $20 \mathrm{q} 24$ \\
\hline SOD1 & $1 q 12.2$ & GLI2 & $2 q 33$ & CSN1S2 & $6 \mathrm{q} 32$ & [TGB1 & 13q13dist & UBE3A & $21 \mathrm{q} 12$ \\
\hline$I F N A R I$ & $I q I 2.2$ & SLC11A1 & $2 q 43$ & PDE6B & $6 q 36$ & $\mathrm{VIM}$ & $13 q 14$ & MEF2A & $21 \mathrm{q} 13$ \\
\hline$T I A M I$ & $1 q 12.2$ & $\mathrm{PAX} 3$ & $2 q 43$ & VAVI & $7 q 15$ prox & RAB18 & $13 q 15 p r o x$ & CHRNA7 & $21 \mathrm{q} 17 \mathrm{dist}$ \\
\hline ATP5O & $1 q 12.2$ & $\mathrm{AK} 2$ & 2q45prox & GM2A & $7 q 21$ & PSMA7 & 13q22prox & GRP58 & $21 q 23-q 24$ \\
\hline GRIKI & $I q I 2.2$ & SMP1* & 2q45prox & HSPA4 & $7 \mathrm{q} 22.1$ & ASIP & 13q22dist & SERPINA3 & 21 q24prox \\
\hline$K C N E 1$ & $\operatorname{lq} I 2.2$ & CRP & $3 q 13$ & RASA1 & $7 \mathrm{q} 25.2$ & ADA & 13q24prox & CHGA & 21q24prox \\
\hline SON & $1 q 12.2$ & \$100A6 & $3 q 21$ & CAST & $7 \mathrm{q} 27$ & CYP11B1 & $14 q 13$ & SERPINA1 & 21q24prox \\
\hline$S L C 5 A 3$ & $1 q 12.2$ & ТНH & $3 q 21$ & TRIM17 & 7 & $\mathrm{TG}$ & $14 q_{15}$ & MITF & $22 \mathrm{q} 22$ \\
\hline CCT8 & $I q I 2.2$ & $I V L$ & $3 q 21$ & ARF1 & 7 & MYC & $14 q 15$ & PBXP] & $22 q 22$ \\
\hline PRSS7 & $1 q 14$ & NGFB & $3 q 23$ & GALT & $8 \mathrm{q} 13$ & $\mathrm{CRH}$ & $14 q^{19}$ & GPX1 & $22 \mathrm{q} 24$ prox \\
\hline POUIF1 & Iq21dist & NRAS & $3 q 23$ & VLDLR & $8 \mathrm{ql}^{17}$ & MMPI & $15 q ! 2$ prox & $\mathrm{HRHI}$ & 22q24dist \\
\hline$G A P 43$ & $\operatorname{Iq} 24$ & TSHB & $3 q 23$ & SFTPC & $8 \mathrm{q} 21$ dist & FDXI & $15 q 21$ prox & GSTAl & 23 q22prox \\
\hline NDUFB4 & lq31prox & UOX & $3 q 31-q 32.1$ & CTSL & $8 \mathrm{q} 25$ & APOA1 & $15 \mathrm{q} 21$ & $\mathrm{BF}$ & $23 q 22$ \\
\hline CASR & $\operatorname{lq} 31$ & ACADM & $3 \mathrm{q} 32.2$ & GSN & $8 \mathrm{q} 28$ & HBB & $15 \mathrm{q} 25$ prox & OLADRB & $23 q 22$ \\
\hline ZNF 148 & $1 \mathrm{q} 31$ & CDC20 & $3 q 35$ & COL9A1 & $9 \mathrm{q} 12.2$ & FSHB & $15 q 25$ dist-q26 & EDNI & 23q24prox \\
\hline UMPS & $1 \mathrm{q} 31$ & COP9* & $3 q 37$ & AMD1 & 9 q16prox & PAX6 & $15 \mathrm{q} 27$ & Fl3Al & 23q24dist \\
\hline HESI & 1q31dist & COL6A3 & $3 q 37$ & $\mathrm{CGA}$ & $9 q 22$ & WT1 & $15 q 27$ & SERPINO1 & 23q24dist \\
\hline CRYGS & $1 \mathrm{q} 33$ & IL6R & 3 & HMGCR & $10 \mathrm{q} 12$ & PGD & $16 q 21$ prox & CYB5 & $24 \mathrm{q} 12$ \\
\hline AHSG & $1 \mathrm{q} 33$ & SAG & 3 & MYH6 & $10 \mathrm{q} 15-\mathrm{q} 21$ & NPPA & $16 \mathrm{q} 21$ & DSG2 & $24 q_{2} 1-q 22$ \\
\hline KNG & $1 q 33$ & HGF & $4 \mathrm{q} 15$ dist $-\mathrm{q} 21$ & MYH7 & $10 q 15-q 21$ & LAMC2 & $16 \mathrm{q} 23$ & ADCYAPI & $24 \mathrm{q} 23$ \\
\hline SIATI & $1 q 33$ & LAMB] & $4 q 22$ & HEXA & $10 \mathrm{q} 15 \mathrm{dist}$ & IL2 & $17 \mathrm{q} 22$ dist & HBA1 & 25q12prox \\
\hline $\mathrm{CP}$ & Iq4 idist & NPY & $4 \mathrm{q} 25-\mathrm{q} 26$ & NP & $10 q 21$ & NOS1 & $17 \mathrm{q} 25$ & EPO & $25 \mathrm{q} 22$ \\
\hline AGTRIB & $1 q 42$ & IGFBP3 & $4 q 26$ & THBS1 & $10 \mathrm{q} 22$ & COMT & $17 \mathrm{q} 26$ & ACTA2 & $26 q 13$ \\
\hline GYG & $1 q 42$ & OPNISW & $4 \mathrm{q} 32$ & MGAT2 & $10 \mathrm{q} 24$ & ELF2 & $17^{\circ}$ & CYP17 & $26 \mathrm{q} 21$ \\
\hline RBPI & $1 \mathrm{q} 43$ & CLCNI & $4 q 34$ & TPM1 & $10 q 26$ & RAB33B & 17 & DNTT & $26 q 21$ \\
\hline NCK 1 & 1943 & SSBP1 & 4q34dist & CYPI9 & $10 \mathrm{q} 31$ & DPEP1 & $18 \mathrm{q} 13$ & PAX2 & $26 q 21$ \\
\hline TFDP2 & 1q43prox & KRTB@ & $5 q 21$ & SORD & $10 \mathrm{q} 32$ & MCIR & $18 q 13$ & $\mathrm{OAT}$ & $26 \mathrm{q} 23$ prox \\
\hline $\mathrm{TF}$ & 1q43dist & AVPR 1A & $5 q 23$ & SPTB & 10q34prox & MT2A & $18 q 15$ & DEFB1 & $27 \mathrm{q} 13$ \\
\hline$M X I$ & lq45prox & IFNG & $5 q 23$ & TGFB3 & $10 \mathrm{q} 34$ dist & GNAO1 & $18 q 15$ & F11 & $27 q^{15}$ \\
\hline$M X 2$ & 1q45prox & IGF1 & 5 q 31 prox & TGMl & $10 \mathrm{q} 34$ & RYR ] & $18 \mathrm{q} 24$ prox & ANK I & $27 q 19$ \\
\hline TFF3 & Iq45prox & FGF6 & $5 \mathrm{q} 35$ prox & TGFA & $11 \mathrm{q} 14$ & PTGIR & $18 \mathrm{q} 24 \mathrm{dist}$ & PLAT & $27 \mathrm{q} 19$ \\
\hline$H M G N I$ & Iq45prox & OSBPL8 & 5 & IL1B & $11 \mathrm{q} 22$ & LHB & 18q24dist & RBP3 & $28 \mathrm{q} 18-\mathrm{q} 19$ \\
\hline CRYAA & lq45med & UGT8 & $6 \mathrm{q} 13$ & CAD & $11 \mathrm{q} 24$ dist & ACACA & $19 \mathrm{ql} 3$ & AGT & $28 q 19$ \\
\hline$C O L 6 A I$ & Iq45med & TXK & $6 \mathrm{q} 14$ & POMC & $11 \mathrm{q} 24$ dist & MYH2 & $19 \mathrm{q} 15-\mathrm{q} 16$ & TYR & $29 q 13$ \\
\hline CBRI & Iq45med & MTP & $6 \mathrm{q} 15$ & ASS & $11 \mathrm{q} 28$ prox & GAS & $19 q_{17}$ & OPCML & $29 q 22$ \\
\hline$R U N X 1$ & Iq45med & HADHSC & 6q15prox & $\mathrm{BRCA} 2$ & $12 \mathrm{q} 15$ & PNMT & $19 \mathrm{q} 17$ & COX8 & $29 q 24$ prox \\
\hline$E R G$ & Iq45dist & GNRHR & $6 \mathrm{q} 32$ prox & SGCG & $12 \mathrm{q} \mid 5$ dist & MAPT & $19 q 22$ prox & LDHA & $29 \mathrm{q} 24$ prox \\
\hline
\end{tabular}

\section{Results and discussion}

Mapping of 195 genes on bovine chromosomes by FISH and SCH analysis

The gene mapping results $(188$ by FISH and seven by $\mathrm{SCH}$ analysis) are summarized in Table 3 . New or refined gene localisations were obtained for all the bovine autosomes although gene density varies among chromosomes. Concerning the distribution of the genes in relation to chromosomal bands, most of those that we have mapped by FISH are located on R-positive bands confirming the general trend that R-positive bands are gene richer than R-negative bands.

\section{Resolving non-concordant chromosome assignments between man and cattle}

At the beginning of our work, we had listed 37 discrepancies (Table 4) between the bovine and human gene maps based on existing comparative data. In order to establish the most accurate bovine/human genome comparison, these discrepancie: were checked to determine whether they were due to true novel synteny groups, to mapping errors in the human or bovine maps or to false orthologous gene pairs. Based on our results (details in Table 4), these discrepancies were sorted into seven classes: (a) Two genes (GUK1 and SAG) belonging to two new synteny groups i.e. between HSA1/BTA7 and HSA2/BTA3; (b) two genes (SKI and PDE1A) originally mismapped on the human map and repositioned following the sequencing of the human genome; (c) five genes (CDC20, FABP3, IVL, COP9, CCT8) mismapped on the bovine map and remapped at expected localizations in this work; (d) eight genes (IL6R, HSPA4, SERPINB1, SSBP1, VAV1, HADHSC, RAB18, PSMA7) originally incorrectly identified and remapped either by FISH or SCH analysis at expected localizations in this work; (e) ten genes (SMP1, GAPDL, GLUL, ACTR2, PABPL1, 
Table 4. BTA/HSA inconsistent localizations examined in this study. Note: localization of underlined genes (see multispecies comparative table) confirms or supports expected localization of neighbouring gene with grey background.

\begin{tabular}{|c|c|c|c|c|c|c|}
\hline Jene $^{a}$ & $\begin{array}{l}\text { HSA } \\
\text { map }\end{array}$ & $\begin{array}{l}\text { MMU } \\
\text { map }\end{array}$ & RNO map & $\begin{array}{l}\text { BTA map } \\
\text { published expected }\end{array}$ & Reference and mapping mode ${ }^{b}$ & BTA localization (this work) when available and comments \\
\hline GUK1 & $1 q 42.13$ & $11 \mathrm{~B} 2$ & $(10 \mathrm{q} 22)$ & $28 / 16$ & $\begin{array}{l}\text { Band et al. ( } 2000) \\
\text { RH (new synteny group confirmed) }\end{array}$ & $\begin{array}{l}\text { No BAC but GUK1 on BTA } 7 \text { defincs a new synteny group } \\
\text { confirmed by the localization of TRIM } 17 \text { and } \underline{\text { ARF1 on BTA7 }(t} \\
\text { work, Table } 3 \text { ) }\end{array}$ \\
\hline SAG & $2 q 37.1$ & $1 \mathrm{C} 5$ & $9 \mathrm{q} 34$ & 3 & $\begin{array}{l}\text { Band et al. ( } 2000 \text { ) } \\
\text { RH (new synteny group confirmed) }\end{array}$ & $\begin{array}{l}\text { SAG assigned to BTA } 3 \text { by SCH and defines a new synteny grou } \\
\text { confirmed by the localization of COP9* and COL } 6 \text { A } 3 \text { on BTA3 } \\
\text { (this work, Table } 3 \text { ) }\end{array}$ \\
\hline SKI & $1 \mathrm{p} 36.32$ & $4 \mathrm{E} 2$ & $(5 q 36)$ & $\begin{array}{l}16 q 21 \\
16 / 33\end{array}$ & $\begin{array}{l}\text { Sonstegard et al. (2000) } \\
\text { F (previously SKI on HSA Iq22. q24) }\end{array}$ & If SKl on HSA lp36.22 (cf UCSC database) OK with BTA16 \\
\hline PDEIA & $2 q 32.1$ & $2 \mathrm{D}$ & $3 q 23$ & $2 / 6$ & $\begin{array}{l}\text { Barendse et al. (1997) } \\
\text { L (previously PDE1A on HSA4) }\end{array}$ & If PDE1A on HSA2 (cf UCSC database) OK with BTA2 \\
\hline $\mathrm{CDC} 20$ & $1 \mathrm{p} 34.2$ & $4 \mathrm{DI}$ & & 3 & Band et al. (2000) RH (mapping error) & CDC20 FISH-mapped to BTA3q35 (this work, Table 3) \\
\hline FABP3 & $1 \mathrm{p} 35.2$ & $4 \mathrm{D} 2.3$ & $5 \mathrm{q} 36$ & 2 & Barendse et al. (1997) L (mapping crror) & FABP3 FISH-mapped to BTA2q45 (pers communication) \\
\hline IVL & $1 \mathrm{q} 21.3$ & $3+2$ & $2 q 34$ & $1941-463$ & $\begin{array}{l}\text { Schmutz el al. (1998) } \\
\text { radioactive ISH (mapping error) }\end{array}$ & IVL FISH-mapped to BTA3q21 (this work, Table 3) \\
\hline COP9* & $2 \mathrm{q} 37.3$ & $(1 \mathrm{C} 5)$ & $(9 q 34)$ & $2 / 3$ & Band et al. (2000) RH (mapping error) & COP9 FISH-mapped to BTA3q37 (this work, Table 3) \\
\hline $\mathrm{CT} 8$ & $21 \mathrm{q} 21.3$ & $16 \mathrm{C} 3.3$ & $(11 \mathrm{q} 22)$ & 7 & Band et al. (2000) RH (mapping error) & CCT8 FISH-mapped to BTA1q12.2 (this work, Table 3) \\
\hline IL6R & $1 \mathrm{q} 22$ & $3 \mathrm{~F} 2$ & $2 \mathrm{q} 34$ & 19 & $\begin{array}{l}\text { Barendse et al. (1999) L (no bovine } \\
\text { scquence so gene identification?) }\end{array}$ & IL6R assigned by SCH to BTA3 (this work, Table 3) \\
\hline HSPA4 & $5 q 31.1$ & $11 \mathrm{~B} 1.3$ & $10 \mathrm{q} 22$ & $3 q 13$ & $\begin{array}{l}\text { Gallagher et at. (1993) FISH (no bovine } \\
\text { sequence but the gene mapped in this } \\
\text { paper is probably HSPA6) }\end{array}$ & HSPA4 FISH-mapped to BTA7q22.1 (this work, Table 3) \\
\hline SERPINOI & $6 \mathrm{p} 25.2$ & $13 \mathrm{~A} 4$ & $17 \mathrm{pl} 2$ & 23 & $\begin{array}{l}\text { Georges et al. ( } 1990) \mathrm{L} \text { (no bovine } \\
\text { sequence so gene identification?) }\end{array}$ & SERPINOI FISH-mapped to BTA23q24 (this work, Tablc 3) \\
\hline SSBP1 & $7 q 34$ & $6 \mathrm{~B} 2$ & $4 q 23$ & 2 & $\begin{array}{l}\text { Barendsc ct al. (1997) L (no bovine } \\
\text { sequencc so gene identification?) }\end{array}$ & SSBP1 FISH-mapped to BTA4q34 (this work, Table 3) \\
\hline VAV1 & $19 \mathrm{pl} 3.3$ & 17E1.1 & $9 q 11-q 12$ & 28 & $\begin{array}{l}\text { Barendsc ct al. (1999) L (no bovine } \\
\text { sequence so gene identification?) }\end{array}$ & VAV1 FISH-mapped to BTA7q15 (this work, Table 3) \\
\hline HADHSC & $4 q 25$ & $3 \mathrm{HI}$ & $2 q 42$ & 26 & $\begin{array}{l}\text { Band et al. (2000) RH (Acc no } \\
\text { AW } 289352 \text { gives } 93 \% \text { sequence } \\
\text { similarity on } 87 \text { bp so gene } \\
\text { identification?) }\end{array}$ & HADHSC FISH-mapped to BTA6q15 (this work, Table 3) \\
\hline
\end{tabular}

$\begin{array}{llllll}\text { RAB18 10p12.1 18A1 (17q12.1) } 9 & & \text { Karall-Albrecht et al, (2000) SCH (Acc no RAB18 FISH-mapped to BTA13q15 (this work, Table 3) } \\ & & & \begin{array}{l}\text { Al461405 gives } 83 \% \text { sequence similarity } \\ \text { on } 184 \text { bp so gene identification?) }\end{array}\end{array}$

PSMA7 20q13.3 2H4 $3 \mathrm{q} 43 \quad 4$ Karall-Albrecht et al. (2000) SCH (Acc no PSMA7 FISH-Inapped to BTA13q22 (this work, Table 3)
AI461430 gives $93 \%$ sequence similarity on 99 bp so gene identification?)

$\begin{array}{lllllll}\text { SMP * } & 1 \mathrm{p} 36.11 & 4 \mathrm{D} 3 & 5 \mathrm{q} 36 & 14 & & \text { Band et al. (2000) } \\ & & & & & 2 & \text { RH (Acc no U89254 in fact := RGS20) } \\ \text { GAPDL } & 2 \mathrm{q} 11.2 & & & 11 & & \text { Barendse et al. (1999) } \\ & & & & & \text { L (gene identification?) }\end{array}$

GLUL 1q25.3 $1 \mathrm{G} 3 \quad$ (13q21) $10 \quad$ Masabanda et al. (1997) GLUL and

16 GLULP FISH-mapped with sequences Acc no Y 10347 (BTA10) and Y10348 (BTA16), respectively

Band et al. (2000)

RH (Acc no U83023 \# ACTR2)
SMP1* FISH-mapped to BT A2q45 (this work, Table 3)

No GAPDL in human database, in fact probably GAPDL 3 on HSA2q11.2 OK with BTA11)

In fact, Y10348 - GLUL (not GLULP) on HSAIq25.3 OK with BTA16q21.

In fact, U83023 = GTF2B on HSA 1 p22.2 OK with BTA3

In fact, U83076 = PABPCl on HSA8q22.3 OK with BTA14

NCAM1 on HSA1 1q23.I OK with BTA15 


\begin{tabular}{|c|c|c|c|c|c|c|}
\hline Gene $^{a}$ & $\begin{array}{l}\text { HSA } \\
\text { map }\end{array}$ & $\begin{array}{l}\text { MMU } \\
\text { map }\end{array}$ & RNO map & $\begin{array}{l}\text { BTA map } \\
\text { published }\end{array}$ & Reference and mapping mode ${ }^{b}$ & BTA localization (this work) when available and comments \\
\hline
\end{tabular}

\begin{tabular}{|c|c|c|c|c|c|c|c|}
\hline $\mathrm{CACNO} 3$ & $12 \mathrm{q} 13$ & $15 \mathrm{~F} 2$ & $7 \mathrm{q} 35$ & 14 & 5 & $\begin{array}{l}\text { Band et al. (2000) } \\
\text { RH (Acc no AW26699] \# CACNO3) }\end{array}$ & In fact, AW266991 = MAFI on HSA8q24.3 OK with BTA14 \\
\hline PLCG2 & $16 \mathrm{q} 23.3$ & $8 \mathrm{El}$ & $19 \mathrm{q} 12$ & 13 & 18 & $\begin{array}{l}\text { Schläpfer et al. (1997) } \\
\text { SCH (Acc no Y00301 \# PLCG2) }\end{array}$ & In fact, Y00301 = PLCG1 on HSA20q12 OK with BTA13 \\
\hline CSH1 & $17 \mathrm{q} 23.3$ & 11E1 & $10 \mathrm{q} 32.1$ & 23 & 19 & $\begin{array}{l}\text { Dietz et al. (1992) } \\
\text { L. (Acc no J02840\# CSHI) }\end{array}$ & In fact, J02840 = PRL on HSA6p22.3 OK with BTA23 \\
\hline MSF & $17 q 23.2$ & $11 \mathrm{E} 2$ & $(10 \mathrm{q} 26)$ & 16 & 19 & $\begin{array}{l}\text { Band et al. (2000) } \\
\text { RH (Acc no AF056218 \# MSF) }\end{array}$ & In fact, AF056218 = PRG4 on IISA Iq31.1 OK with BTA16 \\
\hline NDUFC 1 & $4 \mathrm{q} 31.1$ & $3 \mathrm{D}$ & $(2)$ & 12 & 17 & $\begin{array}{l}\text { Band et al. (2000) RH (membet } \\
\text { identification? of gene family) }\end{array}$ & $\begin{array}{l}\text { NDUFCI FISH-mapped again to BTA } 2 \text { q15 but expected } \\
\text { localization on BTA17 supported by SCH mapping of ELF2 and } \\
\text { RAB33A flanking NDUFC1 (this work, Jable 3) }\end{array}$ \\
\hline NDUFV2 & $18 p+1.2$ & I7E 1.2 & $(9 \mathrm{q} 38)$ & 5 & 24 & $\begin{array}{l}\text { Barendsc et al. (1997) L (member } \\
\text { identification? of gene family) }\end{array}$ & $\begin{array}{l}\text { NDUFV2 FISH-mapped again to BTA5q35 but expected } \\
\text { localization on BTA24 supported by RH mapping of TWSG1 } \\
\text { (AW267141 on BTA24, Band et al, 2000) just below NDUFV2 }\end{array}$ \\
\hline NAP1L1 & $12 \mathrm{q} 21.2$ & $10 D 1$ & $7 \mathrm{q} 21$ & 13 & 5 & $\begin{array}{l}\text { Ma et al. (1998) SCH (member } \\
\text { identification? of gene family or mapping } \\
\text { error?) }\end{array}$ & $\begin{array}{l}\text { No BAC but expected localization on BTA5 supported by SCHI } \\
\text { mapping of OSBPL8 (BTA5, this work Table 3) just below NAPILI }\end{array}$ \\
\hline UBE2I & $16 \mathrm{p} 13.3$ & $17 \mathrm{~A} 3.3$ & $10 \mathrm{q} 12$ & $6 q 34$ & 25 & $\begin{array}{l}\text { Antoniou \& Gallagher (2002) FISH } \\
\text { (member identification? of gene family) }\end{array}$ & $\begin{array}{l}\text { No BAC but cxpected localization on BTA25 supported by RH } \\
\text { mapping of TPSB1 (BTA25, Band et al, 2000) just above UBE2I }\end{array}$ \\
\hline GNAZ & $22 \mathrm{q} 11.2$ & 10B5.3 & $20 \mathrm{p} 12$ & 22 & 17 & $\begin{array}{l}\text { Aleyasin and Barendse (1997) } \\
\text { L. (gene identification?) }\end{array}$ & (FISH of "GNAZ" on BTAI 8q 15 ? in fact = GNAOl) sec tcxt \\
\hline $\mathrm{ACK} 1$ & $3 \mathrm{q} 29$ & $16 \mathrm{~B} 2$ & (1lq11) & 6 & 1 & $\begin{array}{l}\text { Band et al. }(2000) \\
\text { RH (Acc no U96722 = ACK1) }\end{array}$ & ACK1 FISH-mapped to BTA15q23? open question \\
\hline GDH & $1 \mathrm{p} 36.22$ & $(4 \mathrm{E} 1)$ & $(5 q 36)$ & 5 & 16 & $\begin{array}{l}\text { Monteagudo et al. (1992) } \\
\text { Womack ct al. (1986) SCH }\end{array}$ & No bovine scquence, no primers, no BAC, open question \\
\hline NRGN & $11 \mathrm{q} 24.2$ & 9B & $8 \mathrm{q} 21$ & 10 & 15 & $\begin{array}{l}\text { Band et al. }(2000) \\
\text { RH (Acc no } \$ 78295=\text { NRGN) }\end{array}$ & Primers but no BAC, open question \\
\hline FKSGl7* & $8 \mathrm{q} 22.3$ & (4) & (11) & 8 & 14 & $\begin{array}{l}\text { Goldammer et al. }\{2002) \\
\mathrm{SCH}+\mathrm{RH} \text { (gene identification?) }\end{array}$ & No BAC, open question \\
\hline PTGDS & $9 \mathrm{q} 34.3$ & $2 \mathrm{~A} .3$ & $3 \mathrm{P} 13$ & 1 & 11 & $\begin{array}{l}\text { Roncoleta et al. (2002) } \\
\text { SCH \& RH (Acc no AB004647 = } \\
\text { PTGDS) }\end{array}$ & No BAC, open question \\
\hline
\end{tabular}

ADCY2, CACNB3, PLCG2, CSH1, MSF) originally misidentified but for which bovine sequences were available allowing us to recover concordant orthologous human/bovine gene pairs and localizations by BLAST analysis of corresponding accession numbers; (f) four genes (NDUFC1, NDUFV2, NAP1L1, UBE2I) for which close neighbouring genes support their expected localization and not that reported in the literature. These genes are members of large gene families sharing sequence similarities, which may impede identification of true orthologous gene pairs; (g) six genes (GNAZ, ACK1, GDH, NRGN, FKGS17, PTGDS) for which informative data could not be obtained. For the GNAZ gene, we isolated two bovine clones both found located on BTA $18 \mathrm{q} 15$ but verification of the primers used (Table 2 ) revealed that in fact we had selected clones for GNAO1 on HSA16q13 in agreement with the localization on BTA18. Furthermore, Pinton et al. (2000) have shown that the caprine "GNAZ" BAC clone (BTA/CHI22) maps to HSA3p21.3 in agreement with comparative mapping data between BTA22 and HSA3, which suggests that this clone may not contain the GNAZ gene. Finally, the ten genes of classes $(\mathrm{f})$ and $(\mathrm{g})$ were not included in our analysis because comparative mapping data were concordant among man, mouse and rat but not with cattle and we consider that a single gene is not sufficient to support the existence of a novel synteny group. In addition, the existence of paralogs and pseudogenes sharing sequence similarities with a given gene can make it difficult to establish the complete and true comparative chromosome organization among species. Furthermore, as dis- 
cussed by Ozawa et al. (2000) for recently duplicated genes a true orthologous gene may not exist in one of the compared species.

\section{Comparative mapping analysis}

All genes and ESTs mapped to date in cattle, sheep, goat and pig $(2,166)$ were compiled with corresponding data in man, mouse and rat (see Multispecies Comparative Table accessible online at http://locus.jouy.inra.fr/) and a detailed human/cattle/mouse/rat comparative map (Fig. 1) was drawn to propose a direct visualisation of the distribution of conserved chromosomal segments among these four species. Several observations can be derived from our data:

1. The 151 FISH localisations in cattle obtained with caprine BAC clones were all (except one) concordant with those reported in goat by Schibler et al. (1998). This further confirms the high level of genome conservation between the two species and more generally among the three main domestic bovidae, cattle, goat and sheep. It also supports our decision of inferring bovine gene localisations from those mapped only in sheep or goat. Only the COL9A1 gene was found on non-homoeologous bovine and caprine chromosomes i.e. BTA9q12.2 and THI $14 q 11 \rightarrow q 12$. The findings confirm a translocation involving a segment equivalent to the centromeric region of $\mathrm{CHI} 14$ or OAR9 (the ovine counterpart) to the centromeric region of BTA9 during evolution from ancestral chromosomes (Vaiman et al., 1996).

2. Correspondences between human and bovine autosomes proposed on the basis of mapped genes agree nearly completely with previous results obtained by heterologous chromosome painting of bovine chromosomes with human individual paints (Hayes, 1995; Solinas-Toldo et al., 1995; Chowdhary et al., 1996). We show the existence of two new synteny groups i.e. (1) between HSA1q42.13 and BTA7q12 and (2) between HSA2q37.1 $\rightarrow$ q37.3 and BTA3q37. Interestingly, group (1) is supported by comparative mapping data i.e. HSA1/BTA7/ MMU11/RNO10, a combination also found on HSA19p and leads us to question the possibility that the telomeric tip of HSA1q44/MMU11/RNO10 may also be conserved with BTA7. At present, we have not succeeded in isolating BAC clones for this chromosomal segment. Group (2) is located at the telomeric end of both HSA2 and BTA3. These two new synteny groups are particularly interesting since they cover chromosome segments BTA7q12 and BTA3q37 not painted by any human chromosome paint (Hayes, 1995) and situated precisely in pericentromeric and telomeric regions with few known mapped genes. It also suggests that when the correspondence of other such small segments (BTA3q12,4q12 $\rightarrow$ q13, 4q36, 8q12, $25 \mathrm{q} 24,28 \mathrm{q} 12 \rightarrow \mathrm{q} 13$ ) with human chromosomes are determined, it may reveal conserved syntenies unknown up till now.

3. Based on Fig. 1, the coverage of bovine autosomes is estimated at $\sim 76 \%$ if putative segments and centromere interruptions within the same segment are not considered and $\sim 89 \%$ if they are included. Most of the putatively identified conserved regions are associated with R-negative bands known to be gene poor and the empty regions with pericentromeric, telomeric and satellite regions known to be difficult to map and characterize because they contain specific repetitive sequences.

4. Figure 1 shows 84 bovine synteny segments conserved on human autosomes, regardless of gene order and excluding interruptions by human centromeres within the same bovine chromosome. Eight of the 84 conserved segments are supported by only one gene mapped in cattle (Multispecies Comparative Table) but were retained because identical synteny correspondences existed along the given human chromosome suggesting that minor reshuffling between these genome regions had occurred during evolution. These 84 bovine/human conserved segments represent a higher number than those in previous reports ranging from 44 to 58 (Hayes, 1995; Solinas-Toldo et al., 1995; Chowdhary et al., 1996; Iannuzzi et al., 1999; Schibler et al., 1998; Band et al., 2000). This increase in number is partly due to the mosaic organization of human/bovine chromosome synteny as for example between HSA11 and BTA15 and 29. Indeed, painting of bovine chromosomes with HSA11 revealed only two synteny groups (the entire BTA15 and BTA29) while comparison of HSA11 with the bovine genome displays a succession of ten segments conserved with BTA15 and 29 in our work (Fig. 1).

5. Based on the data compiled in the Multispecies Comparative Table and on Fig. 1, it is clear that at this level of resolution, the human genome is much more conserved with that of cattle (and pig) than with that of mouse and rat. However, some variation is observed among chromosomes. For example, HSA 17 and HSA20 are entirely conserved on single chromosomes in the four other species: HSA17/BTA19/MMU11/ RNO10/SSC12 and HSA20/BTA13/MMU2/RNO3/SSC17. In rare cases, the same synteny organisation relative to the human genome is found, as on HSA8p (see Fig. 1) with apparently an identical succession of conserved segments in cattle, mouse, rat i.e. BTA8/27, MMU8/14, RNO16/15, which suggests an ancestral genome organisation maintained in these species. At the other extreme, less than half of one of the smallest human chromosomes i.e. HSA22q11.21 $\rightarrow$ q12.3 shares conserved segments with two bovine or pig chromosomes and with seven different mouse or rat chromosomes revealing a complex pattern relative to HSA22 (Fig. 1).

6. Seventy-eight synteny interruptions were found between human and bovine autosomes (including centromere interruptions). In general, their distribution does not coincide with Rpositive and R-negative band limits except for some instances e.g., along HSAlp, where regions $1 \mathrm{p} 36.33 \rightarrow \mathrm{p} 36.31$, $1 \mathrm{p} 36.13 \rightarrow \mathrm{p} 35.1$ and $1 \mathrm{p} 34.3 \rightarrow \mathrm{p} 11.2$ correspond respectively to parts of BTA16, BTA2 and BTA3. Of the 78 synteny interruptions, 21 are also found in mouse and rat (e.g. see HSA12q23/q24.1, BTA5/17, MMU10C1/5F, RNO7q11/ 12q16) suggesting that they probably occurred before ruminants and rodents diverged. 


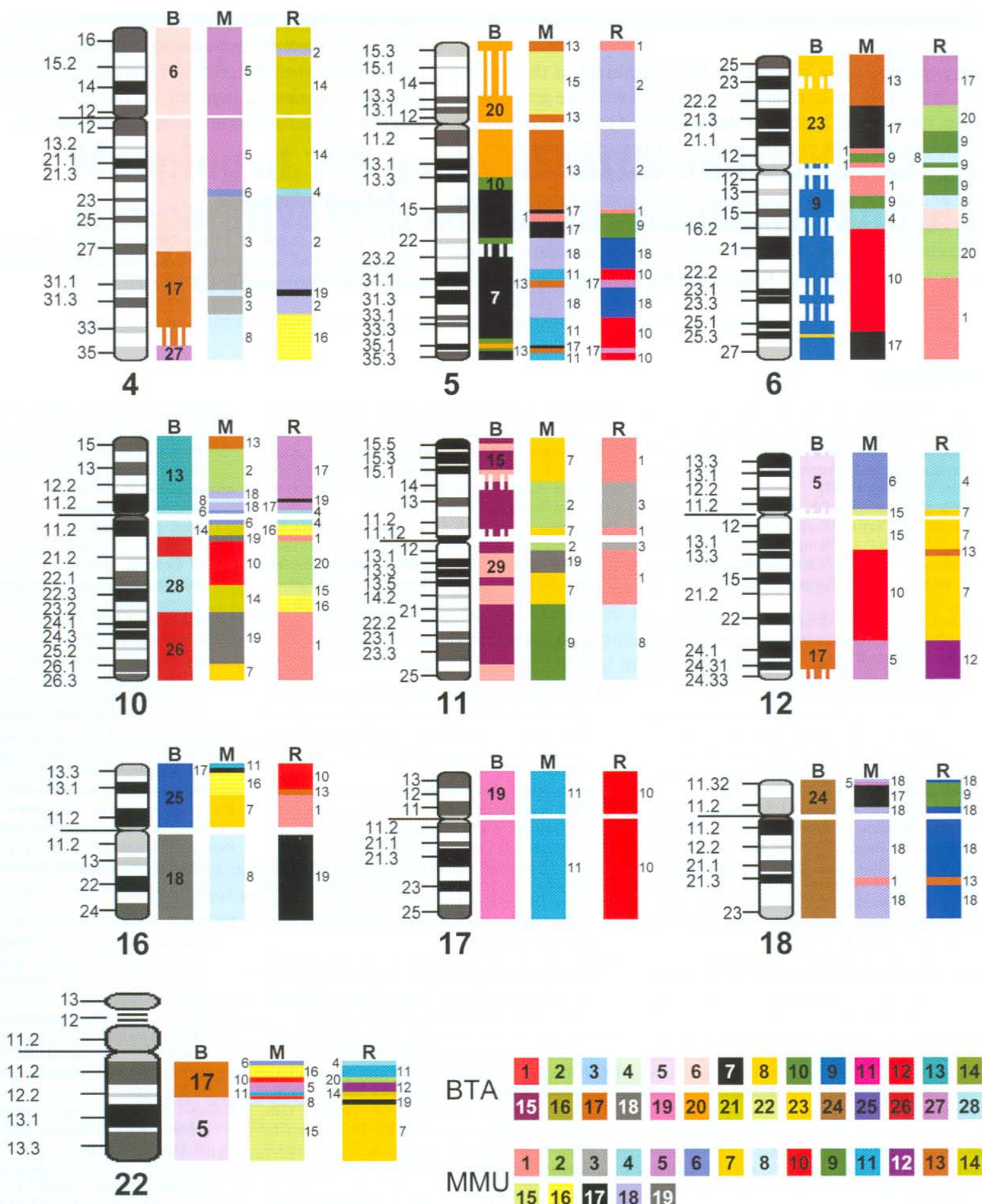

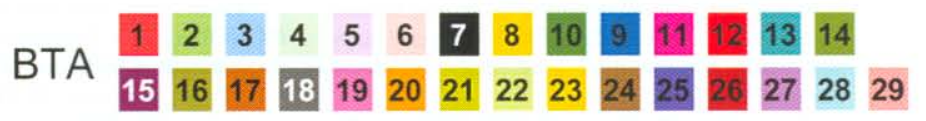

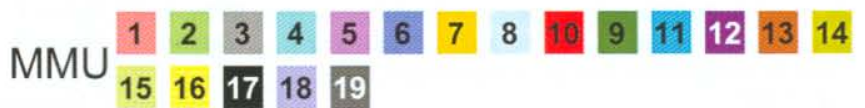

Fig. 1. Distribution and extent of bovine, mouse and rat synteny segments conserved on human autosomes. R-banded human autosomes were taken from the human idiogram at approximately the 550-band level. Bovine (B), mouse ( $\mathbf{M})$ and rat ( $\mathbf{R})$ conserved segments are aligned along each human autosome and represented by color-coded bars with corresponding chromosome numbers on the bars or next to the bars. The color-codes for each of the three species included in the figure i.e. bovine (BTA), mouse (MMU) and rat (RNO) are given at the bottom of the figure. Solid bars correspond to segments confirmed by at least three loci mapped in two or more species (except in a few cases, see text). Unfilled bars correspond to conserved segments between human and bovine genomes deduced from extended comparisons with mouse, rat and pig mapping data. In a few cases, no bar is drawn 
map and order many more genes in bovine chromosomal regions where correspondence with human chromosomes is poorly supported. Extensive radiation hybrid mapping of genes and the complete sequencing of the bovine and pig genomes will provide definitive answers in this direction.

\section{Acknowledgements}

The authors thank M. Pfister-Genskow and L. Doud for technical support with the YAC clones and M. Bertaud and C. Denis for help with the preparation of the bovine BAC DNA.

\section{References}

Alcyasin A, Barendse W: Novel conserved synteny between human chromosome 22 and cattle chromosome 22 established by linkage mapping of transducin alpha-1 subunit (GNAZ). Mammal Genome 8:458-459 (1997).

Amarante MR, Gallagher DS, Burzlaff JD, Lopes CR, Womack JE, Taylor JF, Davis SK: Physical mapping of ADCY2, FSHB and HBB to bovine chromosome 15 by FISH of bovine bacterial artificial chromosome clones. Cytogenet Cell Genet 85:271272 (1999).

Antoniou E, Gallagher D: Chromosomal assignment of the bovine ubiquitin-conjugating enzyme E2I (UBE2I) gene to BTA6q34 defines a new fragment of conserved synteny with human chromosome 16 . Anim Genet 33:388-389 (2002).

Band MR, Larson JH, Rebeiz M, Green CA, Heyen DW, Donovan J, Windish R, Steining C, Mahyuddin P, Womack JE, Lewin HA: An ordered comparative map of the cattle and human genomes. Genome Res 10:1359-1368 (2000).

Barendse W, Vaiman D, Kemp SJ, Sugimoto Y, Armitage SM, Williams JL, Sun HS, Eggen A, Agaba M, Aleyasin SA, Band M, Bishop MD, Buitkamp J, Byrne K, Collins F, Cooper L, Coppettiers W, Denys B, Drinkwater RD, Easterday K, Elduque C, Ennis S, Erhardt G, Li L, et al: A medium-density genetic linkage map of the bovine genome. Mammal Genome 8:21-8 (1997).

Barendse W, Fries R: Genetic linkage mapping, the gene maps of cattle and the lists of loci., in Fries $R$, Ruvinsky A (eds): The Genetics of Cattle (CAB International, Wallingford 1999).

Chowdhary BP, Fronicke L, Gustavsson I, Scherthan $\mathrm{H}$ : Comparative analysis of the cattle and human genomes: detection of ZOO-FISH and gene mapping-based chromosomal homologies. Mammal Genome 7:297-302 (1996).

Dietz AB, Georges M, Threadgill DW, Womack JE, Schuler LA: Somatic cell mapping, polymorphism, and linkage analysis of bovine prolactin-related proteins and placental lactogen. Genomics 14:137143 (1992).

Eggen A, Gautier M, Billaut A, Petit E, Hayes H, Laurent $P$, Urban $C$, Pfister-Genskow $M$, Eilertsen $\mathrm{K}$, Bishop MD: Construction and characterization of a bovine BAC library with four-genome-equivalent coverage. Genet Sel Evol 33:543-548 (2001).

Gallagher DS Jr, Grosz MD, Womack JE, Skow LC: Chromosoma! localization of HSP70 genes in cattle. Mammal Genome 4:388-390 (1993).

Gautier M, Hayes H, Eggen A: An extensive and comprehensive radiation hybrid map of bovine chromosome 15: comparison with human chromosome 11. Mammal Genome 13:316-319 (2002).
Georges M, Lathrop M, Bouquet Y, Hilbert P, Marcotte A, Schwers A, Roupain J, Vassart G, Hanset $R$ : Linkage relationships among 20 genetic markers in cattle. Evidence for linkage between two pairs of blood group systems: B-Z and S-F/V respectively. Anim Genet 21:95-105 (1990).

Goldammer T, Dorroch U, Brunner RM, Kata SR, Womack JE, Schwerin M: Identification and chromosome assignment of 23 genes expressed in meat and dairy cattle. Chrom Res 10:411-418 (2002).

Goureau A, Ycrle M, Schmitz A, Riquet J, Milan D, Pinton $P$, Frelat G, Gellin J: Human and porcine correspondence of chromosome segments using bidirectional chromosome painting. Genomics 36 : 252-262 (1996).

Hayes H: Chromosome painting with human chromosome-specific DNA libraries reveals the extent and distribution of conserved segments in bovine chromosomes. Cytogenet Cell Genet 71:168-174 (1995).

Hayes H, Petit E, Lemieux N, Dutrillaux B: Chromosomal localization of the ovine beta-casein gene by non-isotopic in situ hybridisation and R-banding. Cytogenet Cell Genet 61:286-288 (1992).

Hayes H, Di Meo GP, Gautier M, Laurent P, Eggen A, Iannuzzi L: Localization by FISH of the 31 Texas nomenclature type I markers to both Q- and Rbanded bovine chromosomes. Cytogenet Cell Genet 90:315-320 (2000).

Iannuzzi L, Di Meo GP, Perucatti A, Incarnato D: Comparison of the human with the sheep genomes by use of human chromosome-specific painting probes. Mammal Genome 10:719-723 (1999).

International Human Genome Sequencing Consortium. Nature 409:860-921 (2001).

ISCNDB (2000): International System for Chromosome Nomenclature of Domestic Bovids, Cribiu EP, Di Berardino D, Di Meo GP, Eggen A, Gallagher DS, Gustavsson I, Hayes H, Iannuzzi L, Popescu CP, Rubes J, Schmutz S, Stranzinger G, Vaiman A, Womack J: Cytogenet Cell Genet 92:283-99 (2001).

Karall-Albrecht C, Groenen MA, van der Poel JJ, Barendse W, Womack JE, Kalm E, Looft C: Mapping of 16 ESTs expressed in the bovine mammary gland during lactation. Mammal Genome 11:320$255(2000)$

Laurent P, Elduque C, Hayes H, Saunier K, Eggen A, Leveziel H: Assignment of 60 human ESTs in cattle. Mammal Genome 11:748-754 (2000).

Libert F, Lefort A, Okimoto R, Womack J, Georges M: Construction of a bovine genomic library of large yeast artificial chromosome clones. Genomics 18:270-276 (1993).

Ma RZ, van Eijk MJ, Beever JE, Guerin G, Mummery CL, Lewin HA: Comparative analysis of $82 \mathrm{ex}-$ pressed sequence tags from a cattle ovary cDNA library. Mammal Genome 9:545-549 (1998).
Masabanda J, Wigger G, Eggen A, Stranzinger G, Fries $\mathrm{R}$ : The bovine glutamine synthase gene (GLUL) maps to $10 \mathrm{q} 33$ and a pseudogene (GLULP) to 16q21. Mammal Genome 8:794-795 (1997).

Monteagudo LV, Arruga MV, Tejedor MT, Savva D, Skidmore CJ: Mapping of six genes in cattle using a panel of interspecific hamster $X$ cattle hybrid clones. Anim Genet 23(suppl 1):82 (1992).

Mouse Genome Scquencing Consortium: Initial sequencing and comparative analysis of the mous genome. Nature 420:520-562 (2002).

Ozawa A, Band MR, Larson JH, Donovan J, Green CA, Womack JE, Lewin HA: Comparative organization of cat $t$ le chromosome 5 revealed by comparative mapping by annotation and sequence similarity and radiation hybrid mapping. Proc natl Acad Sci, USA 97:4150-4155 (2000).

Pinton P, Schibler L, Cribiu E, Gellin J, Yerle M: Localization of 113 anchor loci in pigs: improvement of the comparative map for humans, pigs, and goats. Mammal Genome 11:306-315(2000).

Rat genome project: http:/www.hgsc.bcm.tmc.edu/ projects/rat/assembly.html (2003).

Roncoleta M, Kata SR, Womack JE, Amaral ME: Mapping the prostaglandin-D synthase gene (PTGDS) to bovine chromosome 1. Anim Genet 33:322-323 (2002).

Schibler L, Vaiman D, Oustry A, Giraud-Delville C, Cribiu EP: Comparative gene mapping: a fine-scale survey of chromosome rearrangements between ruminants and humans. Genome Res 8:901-915 (1998).

Schläpfer J, Kata SR, Amarante MR, Womack JE: Syntenic assignment of thrombomodulin (THBD) and phosphatidylinositol-specific phospholipase $C$ (PLC-II) to bovine chromosome 13. Anim Genet 28:308-309 (1997).

Solinas-Toldo S, Lengauer C, Fries R: Comparative genome map of human and cattlc. Genomics 27:489-496 (1995).

Sonstegard TS, Garrett WM, Ashwell MS, Bennett GL, Kappes SM, Van Tassell CP: Comparative map alignment of BTA27 and HSA4 and 8 to identify conserved segments of genome containing fat deposition QTL. Mammal Genome 11:682-688 (2000).

Schmutz SM, Moker JS, Berryere TG: In situ hybridisation of five loci to cattle chromosome 1. Cyiogenet Cell Genet 81:51-53(1998).

Vaiman D, Schibler L, Bourgeois F, Oustry A, Amigues Y, Cribiu EP: A genetic linkage map of the male goat genome. Genetics 144:279-305 (1996).

Venter JC, Adams MD, Mycrs EW, Li PW, Mural RJ, Sutton GG, Smith HO, Yandell M, Evans CA, Holt RA, et al: Science 291:1304-1351 (2001).

Womack JE, Moll YD: Gene map of the cow: conservation of linkage with mouse and man. J Hered 77:2$7(1986)$ 